\title{
Avaliação in vitro do efeito de um dentifrício à base de nanohidroxiapatita na rugosidade de superfície do esmalte dental bovino
}

\author{
Effect of nanohydroxyapatite dentifrice on surface roughness of bovine enamel
}

\begin{abstract}
Patrícia Queiroz Rocha dos Reis, ${ }^{1}$ Fernanda Signorelli Calazans, ${ }^{1}$ Luiz Augusto Poubel, ${ }^{1}$ Eduardo Moreira da Silva, ${ }^{2}$ Wesley Veltri Alves, ${ }^{1}$ Marcos de Oliveira Barceleiro ${ }^{1}$

${ }^{1}$ Programa de Pós-Graduação em Odontologia, Curso de Odontologia, Universidade Federal Fluminense, Nova Friburgo, RJ, Brasil

${ }^{2}$ Programa de Pós-Graduação em Odontologia, Curso de Odontologia, Universidade Federal Fluminense, Niterói, RJ, Brasil

- Os autores declaram que não há conflito de interesse.

\section{Resumo}

Objetivo: avaliar in vitro o efeito de um dentifrício à base de nanoHAp na rugosidade superficial do esmalte bovino submetido a ciclagem de $\mathrm{pH}$. Material e Métodos: foram utilizados 12 discos de incisivos bovinos divididos em 2 grupos experimentais: Grupo 1 - dentifrício com flúor - Colgate Total 12 (Colgate-Palmolive Industrial LTDA) e Grupo 2 - dentifrício com nanoHAp - Megasonex (Grupo Goldspire LTDA). Os espécimes foram submetidos ao teste de escovação simulada em 30 ciclos, 2 vezes ao dia, e submetidos a um modelo de ciclagem de pH (desmineralização 6 h pH 4,6/ remineralização 18 h pH 7,0 por dia) durante 14 dias. Todos os espécimes tiveram sua rugosidade superficial avaliada inicialmente e após o tratamento, utilizando um rugosímetro de bancada (Surftest SJ 201). Resultados: na análise estatística pela comparação múltipla entre pares, os testes de Bonferroni e Holm demonstraram que após 7 dias não houve diferença estatisticamente significativa no comportamento dos dentifrícios ( $p>0,05$ ), porém após 14 dias, houve um aumento das médias de rugosidade no grupo 1 (dentifrício com flúor) estatisticamente significativo $(p<0,05)$. Conclusão: 0 dentifrício com nanoHAp promoveu menor rugosidade superficial após 14 dias.

Palavras-chave: Dentifrícios; Esmalte dentário; Hidroxiapatita.

\section{ABSTRACT}

Objective: this study aimed to evaluate in vitro, the effects of dentifrices with hydroxyapatite nanoparticles (nano-HAP) on the roughness of the bovine enamel surface submitted to a pH cycle. Material and Methods: twelve disks of bovine incisors were divided into 2 experimental groups: Group 1, dentifrice with fluoride 1450 ppm-Colgate Total 12 (Colgate-Palmolive Industrial LTDA); Group 2, dentifrice with nano-HAP-Megasonex (Goldspire Group LTDA). The specimens were submitted to 30 cycles of a simulated brushing test, twice a day, and also to a pH cycle model ( $6 \mathrm{~h}$ demineralization at $4.6 \mathrm{pH} / 18 \mathrm{~h} \mathrm{remineralization} 7.0 \mathrm{pH}$ a day) during 14 days. All specimens were evaluated for their superficial roughness at baseline and after the treatment, using a bench rugosimeter (Surftest SJ 201). Results: the statistical analyses by multiple comparison between pairs, using Holm and Bonferroni test's demonstrated that after 7 days no statistically significant difference was observed in the behavior of the dentifrices ( $p>0.01$ ); however, after 14 days, the roughness increased in group 1 (dentifrice with fluoride) and was statistically significant $(p<0.05)$. Conclusion: the dentifrice with nano-HAP promoted less superficial roughness after 14 days.

Keywords: Dental enamel; Hydroxyapatite; Dentifrice.
\end{abstract}

\section{Introdução}

A s estruturas mineralizadas do elemento dental estão sujeitas durante todo o tempo a mudanças de $\mathrm{pH}$ da cavidade bucal. Diante disto, a saliva, que apresenta em sua composição íons de cálcio, fosfato e flúor, auxilia na proteção do esmalte através da formação da película adquirida, agindo diretamente sobre os ácidos presentes, neutralizando-os e fornecendo estes íons para remineralizar lesões cariosas. ${ }^{1}$ Quando a saliva não for capaz de manter o equilíbrio entre a perda e o ganho de íons do esmalte dental, a desmineralização prevalecerá, ${ }^{1,2}$ e para reverter e/ou controlar este processo, substâncias têm sido sugeridas, ${ }^{3}$ dentre as quais destacam-se os compostos à base de flúor, proteínas derivadas do leite como a caseína, e compostos bioativos como as nanopartículas de hidroxiapatita de cálcio, que podem ou não estar associadas ao flúor. ${ }^{4}$

Idealmente, um sistema de remineralização deveria favorecer o ganho de mineral abaixo da superfície ao invés de apenas depositar na camada superficial. Este fato pode explicar a baixa eficácia dos fluoretos em promover o ganho mineral de esmalte e, consequentemente, alterar a cor das lesões de mancha branca. ${ }^{5}$ As pastas contendo hidroxiapatita (HA) possuem algumas vantagens clínicas: eficácia em prevenir cáries, tratamento de doenças periodontais, eliminam os odores bucais e possuem um efeito clareador. $\mathrm{O}$ componente HA na pasta é relatado como sendo efetivo em remover o biofilme dental, promover a remineralização da superfície dos dentes e dificultar o progresso de lesões de cárie incipientes. ${ }^{6}$ Partículas de HA de 20 nanômetros têm uma excelente habilidade de reparar biomimeticamente o esmalte submetido a ataque ácido; ${ }^{7}$ em adição, uma pasta de HA foi desenvolvida para reparar lesões de cárie iniciais, o que demonstrou nanocristais de HA perfeitamente crescidos na interface entre a pasta e o esmalte dental. ${ }^{8}$

A nanohidroxiapatita (nanoHAp) é um dos materiais bioativos mais biocompatíveis. ${ }^{9}$ As nanopartículas de hidroxiapatita têm similaridade com os cristais de HA do esmalte dentário em estrutura morfológica e estrutura cristalina e quando utilizadas para remineralização e tratamento da hipersensibilidade dentinária, associada ao flúor, parecem 
penetrar com maior facilidade no interior das microtrincas em esmalte, promovendo um selamento de qualidade e restaurando a microestrutura e a composição superficial do dente. $^{9}$

Considerando as perspectivas promissoras do uso destas substâncias, o objetivo deste trabalho foi avaliar in vitro o efeito de um dentifrício à base de nanoHAp na rugosidade de superfície do esmalte bovino submetido à ciclagem de $\mathrm{pH}$.

\section{Material e Métodos}

Foram utilizados 12 incisivos bovinos. As amostras ficaram armazenadas em Timol a $0,05 \%$ por até seis meses para não haver degradação de colágeno. A limpeza foi obtida pela raspagem da superfície externa com instrumentos periodontais e profilaxia com pedra pomes e água.

Os dentes foram seccionados em discos de $8 \mathrm{~mm}$ de diâmetro e $2 \mathrm{~mm}$ de espessura, obtidos da área mais plana da porção vestibular de esmalte e ficaram armazenados em água ultrapura a uma temperatura de $4^{\circ} \mathrm{C}$ por até uma semana antes da realização dos ensaios. Os fragmentos foram inicialmente lixados em uma politriz metalográfica (Arotec ${ }^{\circledR}$ modelo-APL-4), empregando lixas de carbureto de silício (lixas d'água) de abrasividade crescente (400, 600, $800,1.200$ e 2.500 ) e com irrigação abundante e, desta forma, foi feita a padronização pela planificação da superfície. Em seguida, foram obtidos os discos a partir do recorte dos fragmentos com o auxílio de pontas diamantadas cilíndricas e turbina de alta rotação, adaptadas em um equipamento padronizador de preparos cavitários. Matrizes de silicone padronizadas para o uso na máquina de escovação simulada foram confeccionadas para cada disco de esmalte bovino.

Todos os espécimes foram avaliados inicialmente a partir de leituras por rugosímetro de superfície (Surftest SJ 201, Mitutoyo, Tóquio, Japão) para obtenção dos dados iniciais. Após a obtenção destes dados iniciais, os espécimes foram divididos em dois grupos experimentais com seis amostras cada: Grupo 1 - dentifrício com 1450 ppm de flúor - Colgate Total 12 (Colgate-Palmolive Industrial LTDA) e Grupo 2 dentifrício com nanoHAp e sem flúor - Megasonex (Grupo Goldspire LTDA), ambos comercialmente disponíveis.

\section{- Tratamento com as Pastas}

Os espécimes foram submetidos ao teste de escovação simulada em uma máquina para teste de escovação modelo MEV-2T, com escovas de cerdas de nylon macias (escova dental Slim Soft - Colgate-Palmolive Industrial LTDA), independentes para cada espécime. $\mathrm{O}$ teste foi realizado com trinta ciclos, duas vezes ao dia, por quatorze dias. Os dentifrícios foram diluídos numa proporção 1:1 (dentifrício/ água destilada), inseridos no dispositivo do equipamento e trocados a cada escovação simulada.

\section{- Ciclagem de pH}

Os espécimes foram tratados com as pastas duas vezes ao dia, antes e após a desmineralização. Os espécimes foram submetidos a um modelo de ciclagem de $\mathrm{pH}$ (desmineralização $6 \mathrm{~h}$ / remineralização $18 \mathrm{~h}$ por dia), durante quatorze dias.

Os espécimes de esmalte foram submetidos ao seguinte modelo de ciclagem de $\mathrm{pH}$ : imersão na solução desmineralizante $[0.1 \mathrm{~mol} / \mathrm{L}$ de ácido lático; $1 \%$ de carboximetilcelulose; $3 \mathrm{mmol} / \mathrm{L}$ de cloreto de cálcio e $1.8 \mathrm{mmol} / \mathrm{L}$ de fosfato monobásico de potássio $\mathrm{pH} \cong 4,6]$ por 6 horas diárias e imersão na solução remineralizante $[1,5 \mathrm{mmol} / \mathrm{L}$ de nitrato de cálcio tetrahidratado, $0,9 \mathrm{mmol} / \mathrm{L}$ fosfato de sódio dibásico di-hidratado, $150 \mathrm{mmol} / \mathrm{L}$ de cloreto de potássio tampão/L de Tris 0,02 mol, 0,05 ppm de flúor, $\mathrm{pH} \mathrm{7,0]} \mathrm{por} 18$ horas. Foram utilizados $30 \mathrm{ml}$ por espécime, numa temperatura de $37^{\circ} \mathrm{C}$, sem agitação. Os espécimes foram tratados com as pastas, conforme descrito anteriormente, antes e após o ciclo de desmineralização. ${ }^{10}$

\section{- Rugosidade}

Todos os espécimes tiveram a rugosidade de superfície avaliada, inicialmente e após o tratamento, utilizando um rugosímetro de superfície (Surftest SJ 201, Mitutoyo, Tóquio, Japão). Quatro traços de rugosidade espaçados em $120^{\circ}$, com um percurso de $0,25 \mathrm{~mm}$ e uma velocidade de $0,1 \mathrm{~mm} / \mathrm{s}$, foram registrados para cada amostra, e a média da rugosidade da superfície $(\mathrm{Ra}-\mu \mathrm{m})$ foi determinada. O parâmetro Ra foi obtido utilizando a seguinte fórmula:

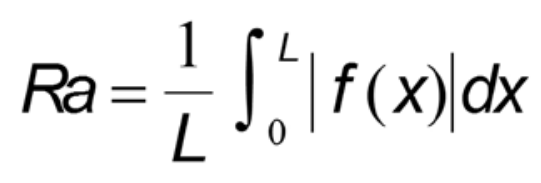

Onde $L$ é o comprimento da secção e $f(x)$ é a função de deslocamento.

\section{- Análise Estatística}

Os valores de rugosidade foram estatisticamente tratados por Anova e posteriormente pelo teste de Comparação múltipla entre pares de Bonferroni e Holm, utilizando-se o software SigmaPlot versão 11.0 (Systat Software).

\section{Resultados}

A tabela 1 apresenta os valores de Ra em cada amostra obtidos nos diferentes grupos, nos diferentes períodos de avaliação. 
Tabela 1. Resultado do teste de rugosidade (médias de Ra e desvio padrão (DP) da média)

\begin{tabular}{c|c|c|c|c|c|c}
\hline & \multicolumn{2}{|c|}{ Baseline } & \multicolumn{2}{c}{ 7 dias } & \multicolumn{2}{c}{ 14 dias } \\
\hline Grupo 1 & Média & DP & Média & DP & Dédia & 0,01 \\
\hline Dente 1 & 0,10 & 0,01 & 0,29 & 0,03 & 0,71 & 0,01 \\
\hline Dente 2 & 0,13 & 0,02 & 0,61 & 0,08 & 1,02 & 0,01 \\
\hline Dente 3 & 0,12 & 0,03 & 0,31 & 0,02 & 0,69 & 0,11 \\
\hline Dente 4 & 0,11 & 0,03 & 0,26 & 0,03 & 0,61 & 0,03 \\
\hline Dente 5 & 0,11 & 0,01 & 0,24 & 0,01 & 0,46 & 0,04 \\
\hline Dente 6 & 0,11 & 0,04 & 0,45 & 0,03 & 0,97 & DP \\
\hline & & & & & & 0,01 \\
\hline Grupo 2 & Média & DP & Média & DP & Média & 0,02 \\
\hline Dente 7 & 0,09 & 0,02 & 0,26 & 0,01 & 0,40 & 0,01 \\
\hline Dente 8 & 0,10 & 0,02 & 0,21 & 0,03 & 0,35 & 0,03 \\
\hline Dente 9 & 0,09 & 0,02 & 0,22 & 0,01 & 0,31 & 0,01 \\
\hline Dente 10 & 0,09 & 0,01 & 0,27 & 0,01 & 0,55 & 0,04 \\
\hline Dente 11 & 0,13 & 0,02 & 0,55 & 0,06 & 0,56 & \\
\hline Dente 12 & 0,12 & 0,02 & 0,23 & 0,02 & 0,85 & \\
\hline
\end{tabular}

Já na tabela 2, podem ser observados os valores médios de Ra nos diferentes grupos testados.

Tabela 2. Média de valores de rugosidade dos grupos testados

\begin{tabular}{c|c|c|c|c|c|c}
\hline & \multicolumn{2}{|c|}{ Baseline } & \multicolumn{2}{c}{$\mathbf{7}$ dias } & \multicolumn{2}{c}{14 dias } \\
\hline Grupo 1 & Média & DP & Média & DP & Média & DP \\
\hline & $0,11^{\mathrm{a}}$ & 0,01 & $0,36^{\mathrm{b}}$ & 0,14 & $0,74^{\mathrm{c}}$ & 0,21 \\
\hline Grupo 2 & Média & DP & Média & DP & Média & DP \\
\hline & $0,10^{\mathrm{a}}$ & 0,02 & $0,29^{\mathrm{b}}$ & 0,13 & $0,50^{\mathrm{b}}$ & 0,20 \\
\hline
\end{tabular}

Obs.: Letras diferentes significam diferença estatística

$\mathrm{Na}$ análise estatística pela comparação múltipla entre pares (Tabela 2), os testes de Bonferroni e Holm demonstraram um aumento significativo da rugosidade superficial de todas as médias de Ra após 7 e 14 dias de tratamento ( $\mathrm{p}<$ 0,01 ), quando comparadas com as médias de Ra no baseline. E na comparação entre os grupos de tratamento, após 7 dias não houve diferença estatisticamente significativa no comportamento dos dentifrícios ( $\mathrm{p}>0,05$ ), porém após 14 dias, houve um aumento das médias de Ra no grupo 1 (dentifrício convencional) estatisticamente significativo $(\mathrm{p}<0,05)$.

\section{Discussão}

O esmalte dental é uma estrutura mineralizada, formada principalmente por cristais de hidroxiapatita que, dependendo das condições do meio, como, por exemplo, acúmulo de biofilme, higiene deficiente e substrato favorável, o desequilíbrio entre a perda e ganho de minerais prevalece. A redução da concentração mineral é devido à queda de $\mathrm{pH}$ abaixo do nível crítico para dissolução da hidroxiapatita. ${ }^{11}$

Com o objetivo de reproduzir e simular a perda e ganho de minerais, ou seja, desmineralização e remineralização, que ocorre na cavidade bucal, lesões artificiais são recriadas nos estudos in vitro. ${ }^{12} \mathrm{Em}$ sua maioria, são compostas por ácido lático ou acético e permitem a formação de lesões de cárie incipientes. ${ }^{13}$ Portanto, foi utilizado neste estudo um desafio desmineralizante (solução com ácido lático) simulando alto desafio de cárie e remineralizante com os dentifrícios e imersão em solução neutra. O objetivo foi simular duas escovações e seis refeições diárias - o total foi de 6 horas de $\mathrm{pH}$ crítico em torno de 4,0 e 18 horas de $\mathrm{pH}$ neutro 7,0. ${ }^{14}$

Quando essas lesões de cárie ainda estão em estágio incipiente, clinicamente identificadas como manchas brancas, terapias remineralizadoras podem reverter seu progresso, além de reparar essas lesões. ${ }^{1}$ Para tanto, dentifrícios com fluoretos são o protocolo convencional para prevenção da formação dessas lesões e para remineralização. A presença constante de qualquer íon flúor na cavidade oral possibilita ao flúor uma atuação considerada anticárie. ${ }^{15}$ Em contrapartida, qualquer $\mathrm{F}$ absorvido pelo organismo e, consequentemente, circulante através da corrente sanguínea possui potencial de manifestar alguns efeitos colaterais. Estes efeitos colaterais são dependentes da dose absorvida e também do tipo de exposição ao fluoreto. A toxicidade causada pelo íon F pode ser dividida em aguda e crônica, ambos sendo efei- 
tos sistêmicos do flúor. Os sintomas da toxicidade aguda, que ocorre quando uma grande dose de flúor é ingerida de uma só vez, incluem vômitos, mal estar gástrico, hipersalivação, suor frio, queda de pressão, arritmia cardíaca, desorientação, coma e até mesmo a morte do indivíduo. ${ }^{15}$ A toxicidade crônica pelo íon flúor se manifesta como fluorose dental, que é uma alteração do esmalte que acontece durante o período de formação dos dentes como consequência da ingestão excessiva de fluoretos por um período de tempo prolongado. Contemporaneamente, a fluorose dentária decorre da ingestão de fluoretos através de múltiplas fontes de flúor, sendo estas geralmente a água e dentifrícios fluoretados. ${ }^{16}$

Além disso, concentrações altas de flúor podem limitar a remineralização da subsuperfície de esmalte desmineralizada, já que causam rápida precipitação mineral na superfície do esmalte e obstrução dos poros da superfície do esmalte. Este fato pode explicar a baixa eficácia dos fluoretos em promover o ganho mineral de esmalte e, consequentemente, alterar a cor das lesões de mancha branca. ${ }^{5}$

Considerando as limitações supracitadas em relação à utilização de fluoretos, torna-se importante a busca por compostos bioativos alternativos ao flúor que sejam uma opção viável de utilização pela população e que possam ser seguramente incorporados aos produtos de uso odontológico, como nos dentifrícios, sem oferecer os riscos de toxicidade pertencentes ao íon flúor. Portanto, o objetivo deste trabalho foi avaliar in vitro o efeito de um dentifrício com nanohidroxiapatita na rugosidade de superfície do esmalte bovino submetido à ciclagem de $\mathrm{pH}$ por meio de teste de rugosidade de superfície, simulando alto desafio cariogênico, comparando-o a um dentifrício convencional com flúor.

As características físicas e ópticas da superfície de esmalte nos indicam atividade ou não das lesões iniciais de cárie. Lesões de cárie ativa são descritas como manchas brancas, opacas e rugosas. Posteriormente, com a adequação da higiene oral, essas manchas se tornam inativas com aspecto liso e brilhoso. Clinicamente, o diagnóstico dessas lesões se dá a partir da observação visual da superfície (opaca ou brilhosa) e tátil, com relação à textura superficial (lisa ou rugosa). ${ }^{17}$ A partir desse conceito, foi escolhido o teste de rugosidade de superfície para a avaliação da superfície de esmalte tratada nesse estudo, se aproximando da realidade clínica de diagnóstico de lesões iniciais de cárie e processo de remineralização. Segundo Field et al. ${ }^{18}$ apesar de vários métodos disponíveis para avaliação in vitro das mudanças de superfície de esmalte, o teste de rugosidade de superfície ainda é o principal e mais reportado nos estudos.
Os resultados desse estudo demonstraram que a rugosidade de superfície do esmalte submetido à ciclagem de $\mathrm{pH}$ e tratamento com dentifrício convencional com flúor foi maior do que com o tratamento com dentifrício experimental com nanoHAp, após quatorze dias. O que sugere um processo de reversão da desmineralização, ou seja, uma maior remineralização superficial do esmalte tratado com nanoHAp, demonstrado por uma maior lisura superficial. Concordando assim com as recomendações de uso pelo fabricante, de no mínimo quatorze dias para que haja esse processo de remineralização. Este estudo está de acordo com Tschoppe et al..$^{19}$ que encontraram que cremes dentais que contenham nanoHAp revelaram efeitos remineralizantes mais elevados em comparação com dentifrícios fluoretados (AmF) em dentina bovina e tendências comparáveis foram obtidas para o esmalte. Além disso, Souza et al. ${ }^{13}$ utilizaram uma pasta experimental à base de nanoHAp e encontraram resultados que sugeriram a redução da desmineralização na dentina e um aumento na remineralização do esmalte.

Em contrapartida, Comar et al. ${ }^{20}$ avaliaram in vitro o potencial de pastas experimentais contendo nanopartículas de hidroxiapatita a $10 \%$ e $20 \%$ (nanoHAp), com ou sem fluoreto, na prevenção da desmineralização dentária e concluíram que as pastas experimentais nanoHAp, independentemente da presença ou não de fluoreto, não foram capazes de reduzir a desmineralização dentária in vitro. Há de se ressaltar, no entanto, que no trabalho de Comar et al. ${ }^{20}$ os autores fizeram a avaliação por meio de teste de dureza longitudinal, não permitindo assim a comparação com os resultados obtidos neste estudo.

Vale ressaltar que este estudo foi realizado in vitro e, apesar da metodologia tentar se aproximar ao máximo do que ocorre na cavidade bucal, ela não corresponde exatamente à realidade clínica. Com isso, sugere-se a continuidade novos estudos para avaliar a real eficácia desses materiais, realizando-se outros testes, tais quais as avaliações de cor e brilho, microdureza, imagens em MEV e contagem de elementos $\mathrm{Ca}$ e P.

Devido aos resultados promissores desta pesquisa sugerimos que a nanohidroxiapatita deve continuar sendo estudada, com estudos complementares in situ e in vivo para melhor elucidar os efeitos desse composto em outras propriedades além da rugosidade superficial, assim como a realização de outras avaliações e testes.

\section{Conclusão}

Conclui-se que o dentifrício experimental à base de nanoHAp promoveu uma menor rugosidade do que o 
convencional com flúor após 14 dias de tratamento sob condições de ciclagem de $\mathrm{pH}$, sugerindo um aumento no processo de remineralização superficial do esmalte.

O presente estudo mostra-se de extrema importância para a comunidade científica por encontrar resultados bastante promissores em relação à nanohidroxiapatita, um composto bioativo e biocompatível que pode ser utilizado como alternativa aos fluoretos nos dentifrícios. Este estudo sugere que dentifrícios contendo nanohidroxiapatita possuem um potencial remineralizador considerável e inclusive maior do que os dentifrícios convencionais fluoretados, podendo ser uma excelente alternativa aos dentifrícios contendo flúor, considerando-se as limitações da utilização deste elemento, que já foram claramente descritas na literatura.

\section{Referências}

1. Featherstone JDB. Dental caries: a dynamic disease process. Aust Dent J. 2008 2. : Santos; 2005. P. 71-96.

3. García-Godoy

4. F, Hicks MJ. Maintaining the integrity of the enamel surface: The role of dental biofilm, saliva and preventive agents in enamel demineralization ; 53(3):286-91.

5. Fejerskov O, Nyvad B, Kidd E. Caracterísitcas clínicas da cárie dentária. In: Fejerskov O, Kidd E, editores. Cárie dentária: a doença e seu tratamento clínico. São Paulo and remineralization. J Am Dent Assoc. 2013;139,Suppl.2:S25-34.

6. Brambilla E. Fluoride-Is It Capable of Fighting Old and New Dental Diseases? Caries Res. 2001;35,Suppl.1:S6-9.

7. Huang S, Gao S, Cheng L, Yu H. Combined effects of nano-hydroxyapatite and Galla chinensis on remineralisation of initial enamel lesion in vitro. J Dent. 2010;38(10):811-9.

8. Niwa M, Sato T, Li W, Aoki H, Aoki H, Daisaku T. Polishing and whitening properties of toothpasete containing hydroxyapatite. J Mater Sci Mater Med. 2001;12(3):277-81.

9. Huang SB, Gao SS, Yu HY. Effect of nano-hydroxyapatite concentration on remineralization of initial enamel lesion in vitro. Biomed Mater. 2009;4:034104. 10. Yamagishi Y, Onuma K, Suzuki T, Okada F, Tagami J, Otsuki M, et al. A synthetic enamel for rapid tooth repair. Nature. 2005;433(7028):819.

11. Zhao H, He W, Wang Y, Zhang X, LiZ, Yan S, et al. Biomineralization of large hydroxyapatite particles using ovalbumin as biosurfactant. Mater Letters. 2008;62(20):3063-5.

12. Magalhães AC., et al. Comparison of cross-sectional hardness and transversal microradiography of artificial carious lesions induced by different demineralizing solutions and gels. Caries Res. 2009;43(6):474-83.

13. Soares LES, De Carvalho Filho ACB. Protective effect of fluoride varnish and fluoride gel on enamel erosion: roughness, SEM-EDS, and $\mu$-EDXRF studies. Microsc Res Tech. 2015;78(3):240-8.

14. Ten Cate JM, Dundon KA, Vernon PG, Damato FA, Huntington E, Exterkate RAM, et al. Preparation and measurement of artificial enamel lesions, a four-laboratory ring test. Caries Res. 1996;30(6):400-7.

15. Souza BM, Comar LP, Vertuan M, Fernandes Neto C, Buzalaf MAR, Magalhães AC. Effect of an experimental paste with hydroxyapatite nanoparticles and fluoride on dental demineralisation and remineralisation in situ. Caries Res. 2015;49(5):499-507.

16. Da Silva EM, De Sá Rodrigues CUF, Dias DA, Da Silva S, Amaral CM, Guimarães JGA. Effect of Toothbrushing-mouthrinse-cycling on Surface Roughness and Topography of Nanofilled, Microfilled, and Microhybrid Resin Composites. Oper Dent. 2014;39(5):521-9.

17. Cury JA, Tenuta LMA. Evidências para o uso de fluoretos em odontologia. Odontologia baseada em evidências. 2010;2(4):1-20.

18. Narvai PC, Antunes JLF, Frias AC, Soares MC, Marques RAA, Teixeira DSC, et al. Fluorose dentária em crianças de São Paulo. Rev Saúde Pública. 2013;47. Suppl. 3:S148-53.

19. Ando M, Eckert GJ, Zero DT. Preliminary study to establish a relationship between tactile sensation and surface roughness. Caries Res. 2010;44(1):24-8.

20. Field J, Waterhouse P, German M. Quantifying and qualifying surface changes on dental hard tissues in vitro. J Dent. 2010;38(3):182-90.

21. Tschoppe P, Zandim DL, Martus P, Kielbassa AM. Enamel and dentine remineralization by nano-hydroxyapatite toothpastes. J. Dent. 2011;39(6):430-7.

22. Comar LP, Souza BM, Gracindo LF, Buzalaf MA, Magalhães AC. Impact of experimental Nano-HAP Pastes on Bovine Enamel and Dentine Submitted to a pH cycling Model. Braz Dent J. 2013; 24(3):273-8.

\section{Mini Currículo e Contribuição dos Autores}

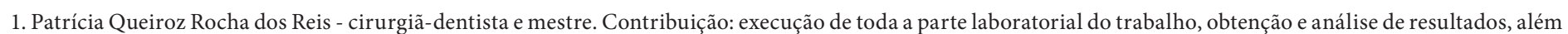
do desenvolvimento da parte escrita.

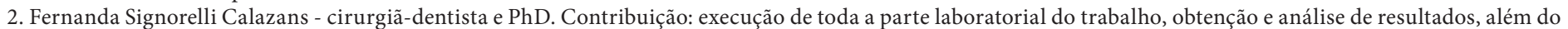
desenvolvimento da parte escrita.

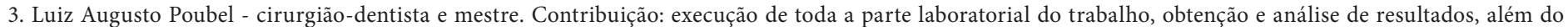
desenvolvimento da parte escrita.

4. Eduardo Moreira da Silva - cirurgião-dentista e PhD. Contribuição: orientação de todas as etapas, correção e finalização da parte escrita.

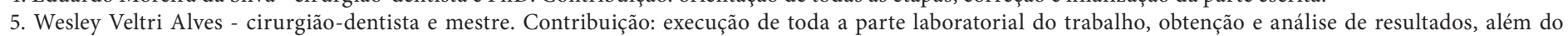
desenvolvimento da parte escrita.

6. Marcos de Oliveira Barceleiro - cirurgião-dentista e PhD. Contribuição: orientação de todas as etapas, correção e finalização da parte escrita.

Recebido em: 04/04/2017 / Aprovado em: 18/05/2017

Autora Correspondente

Fernanda Signorelli Calazans

E-mail: fecalazans@yahoo.com.br 